\title{
La asociación entre la resistencia a la insulina y la rigidez arterial en pacientes mexicanos sin diabetes mellitus tipo 2
}

\author{
Roopa Mehta ${ }^{1,2 *}$, Neftali E. Antonio-Villa1,3, Omar Y. Bello-Chavolla4, Alexandro J. Martagón ${ }^{1,5}$, \\ Daniel Elias-López ${ }^{1,2}$, Arsenio Vargas-Vázquez1,3, Donaji V. Gómez-Velasco', Paloma Almeda-Valdés',2, \\ Liliana Muñoz-Hernández ${ }^{1,2}$, Ivette Cruz-Bautistat, y Carlos A. Aguilar-Salinas, ${ }^{1,2,5}$ \\ ${ }^{1}$ Unidad de Investigación de Enfermedades Metabólicas, Instituto Nacional de Ciencias Médicas y Nutrición Salvador Zubirán; ${ }^{2}$ Departamento de \\ Endocrinología y Metabolismo, Instituto Nacional de Ciencias Médicas y Nutrición Salvador Zubirán; ${ }^{3}$ Facultad de Medicina, Universidad Nacional \\ Autónoma de México; ${ }^{4}$ División de Investigación, Instituto Nacional de Geriatría; ${ }^{5}$ Escuela de Medicina y Ciencias de la Salud, Tecnologico de \\ Monterrey. Ciudad de México, México
}

\section{Resumen}

Antecedentes: La rigidez arterial central aórtica (RACA) es un factor de riesgo cardiovascular independiente. La resistencia a la insulina (RI) contribuye al riesgo asociado a RACA. Objetivo: Evaluar la asociación entre RI y RACA en una población mexicana sin diabetes. Métodos: La RI se estimó con HOMA2-IR y (Homeostatic Model Assessment 2-Insulin Resistance) otros subrogados (METS-IR [Metabolic score for IR], QUICKI [Quantitative Insulin Sensitivity Check Index], TyG [ratio triglicéridos/glucosa], TyG*IMC [TyG*índice de masa corporal] y TG/HDL [ratio TG/lipoproteínas de alta densidad]). Se evaluó la RACA mediante el análisis de velocidad de onda del pulso carotídeo-femoral (VOP ) y el índice de aumentación estandarizado (Al-75). Se realizaron correlaciones bivariante entre los subrogados y la VOP ${ }_{\text {cf }}$ RACA aumentada se definió como VOP ${ }_{c f}$ arriba del percentil 90. Se obtuvieron puntos de corte y área bajo la curva $(A B C)$ para cada subrogado para estimar RACA aumentada. Resultados: Se incluyó 358 pacientes. Se encontró una correlación entre HOMA2-IR y VOP ; esta correlación se replicó con Ios subrogados. METS-IR y TyG*IMC tuvieron el mayor grado de correlación con VOP ${ }_{\text {cf }}$ Al ajustar, las correlaciones con TyG*IMC, METS-IR, HOMA2-IR y QUICKI mantuvieron significancia. La correlación con Al-75 fue mayor para HOMA2-IR. METSIR y TyG mostraron la mejor ABC. Los pacientes con prediabetes tuvieron mayor VOP ${ }_{\text {cf }}$ Conclusiones: La relación entre la RI y la RACA está presente desde etapas no diabéticas; esta asociación puede conllevar mayor riesgo cardiovascular.

PALABRAS CLAVE: Resistencia a la insulina. Rigidez arterial. Prediabetes. Perfil cardiometabólico.

\section{Association between insulin resistance and arterial stiffness in Mexican patients without type 2 diabetes}

\section{Abstract}

Introduction: Central aortic arterial stiffness (CAAS) is an independent cardiovascular risk factor. Insulin resistance (IR) contributes to CAAS-associated risk. Objective: To evaluate the association between IR and CAAS in a Mexican population without diabetes. Methods: IR was estimated with Homeostatic Model Assessment 2-Insulin Resistance (HOMA2-IR) and other surrogate markers (Metabolic score for IR [METS-IR], Quantitative Insulin Sensitivity Check Index [QUICKI], triglycerides/ glucose index [TyG], TyG*body mass index [TyG*BMI] and triglycerides/high-density lipoprotein cholesterol ratio [TG/HDL-C]). CAAS was evaluated using carotid-femoral pulse wave velocity analysis $\left(P W V_{c f}\right)$ and the standardized augmentation index

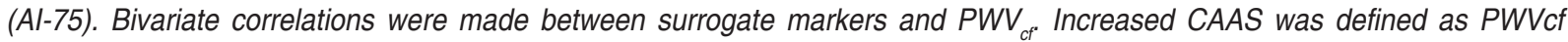
above the 90th percentile. Thresholds and area under the curve (AUC) were obtained for each surrogate marker in order

Correspondencia:

*Roopa Mehta

E-mail: roopamehta@yahoo.com
Gac Med Mex. 2021;157:539-547

Disponible en PubMed

www.gacetamedicademexico.com

0016-3813/@ 2021 Academia Nacional de Medicina de México, A.C. Publicado por Permanyer. Este es un artículo open access bajo la licencia CC BY-NC-ND (http://creativecommons.org/licenses/by-nc-nd/4.0/). 
evaluate their performance in estimating increased CAAS. Results: Three hundred and fifty-eight patients were included. $A$ correlation was found between HOMA2-IR and PWV ${ }_{c f}$; this correlation was replicated with other surrogate markers. METS-IR and TyG*IMC had the highest degree correlation with PWVcf. When adjustments were made for covariables, the correlations with TyG*IMC, METS-IR, HOMA2-IR and QUICKI maintained significance. HOMA2-IR showed the strongest correlation with Al-75. METS-IR and TyG showed the best AUC. Patients with prediabetes had the highest PWVcf. Conclusions: The relationship between IR and CAAS is present before the onset of diabetes; this association may entail higher cardiovascular risk.

KEY WORDS: Insulin resistance. Arterial stiffness. Prediabetes. Cardiometabolic diseases.

\section{Introducción}

La resistencia a la insulina $(\mathrm{RI})$ se caracteriza por una disminución en la sensibilidad de los tejidos a la insulina. Está asociado con la diabetes tipo 2 (DT2), la obesidad y el síndrome metabólico; este parámetro contribuye al riesgo cardiovascular (CV) asociado a estas enfermedades ${ }^{1-3}$. Los mecanismos fisiopatológicos involucrados en la relación entre la RI y la morbimortalidad CV no son bien conocidos ${ }^{4}$. Algunos investigadores han explorado una asociación entre la $\mathrm{RI}$ y la rigidez arterial central-aórtica (RACA) ${ }^{5}$.

El sistema arterial mantiene un flujo sanguíneo adecuado y funciona como un amortiguador ${ }^{6}$. Esto depende de la elasticidad de las arterias. Las arterias pueden perder su distensibilidad vascular con la edad y con la hipertensión arterial, enfermedad renal crónica, DT2 y aterosclerosis ${ }^{7}$. La RACA es un factor de riesgo CV independiente ${ }^{8,9}$. Cuando una arteria es rígida, la onda de pulso hacia adelante es más rápida y se refleja de la periferia más rápidamente, llegando al corazón durante la sístole temprano, produciendo un aumento en la presión arterial sistólica; al mismo tiempo, la presión diastólica se reduce y existe una reducción en la perfusión coronaria diastólica ${ }^{10}$, además de la transmisión de más energía pulsátil hacia las arterias pequeñas, produciendo daño microvascular.

El estándar de oro para medir la sensibilidad a la insulina es la pinza euglucémica-hiperinsulinémica"11. Este método no es útil en la práctica clínica por ser laborioso, invasivo y $\mathrm{caro}^{12}$. Un índice subrogado ampliamente utilizado para estimar la $\mathrm{Rl}$ es el HOMA2-IR (Homeostatic Model Assessment for Insulin Resistance). Otros índices como el QUICKI (Quantitative Insulin Check Index), y subrogados que utilizan parámetros metabólicos y antropométricos en sus fórmulas (p. ej., METS-IR [Metabolic score for IR], QUICKI [Quantitative Insulin Sensitivity Check Index], TyG [ratio triglicéridos/glucosa], TyG*IMC [TyG*índice de masa corporal] y TG/HDL [ratio TG/lipoproteínas de alta densidad]) $)^{13-15}$ también tienen una buena correlación con el método de pinza.

La velocidad de onda del pulso carotídeo-femoral $\left(V_{\mathrm{V}} \mathrm{P}_{\mathrm{cf}}\right)$ es el estándar de oro para la evaluación no invasiva de la $\operatorname{RACA}^{16,17}$. El papel que juega la $R I$ en promover un aumento RACA no ha sido esclarecido. Además, esta relación puede estar sesgada a la suma de alteraciones metabólicas vistas en pacientes con DT2. El objetivo de este estudio es evaluar la asociación entre la RI y la RACA $\left(\mathrm{VOP}_{\mathrm{cf}}\right)$ en una población mexicana sin diabetes.

\section{Metodología}

\section{Diseño y población de estudio}

Se realizó un estudio transversal reclutando sujetos entre enero 2017 y diciembre 2020 en la Unidad de Investigación de Enfermedades Metabólicas del Instituto Nacional de Ciencias Médicas y Nutrición Salvador Zubirán. La edad de los participantes estuvo entre 18 y 70 años, y no tenían diagnóstico previo de DT2. Se incluyeron pacientes con diagnósticos previos de prediabetes (glucosa en ayuno 100-125 mg/dl o hemoglobina glucosilada [HbA1c] 5.7-6.4\%), hipertensión arterial sistémica (diagnostico por presión arterial $\geq 140 / 80 \mathrm{mmHg}$ y/o con tratamiento con antihipertensivos), obesidad (IMC $\geq 30 \mathrm{~kg} / \mathrm{m}^{2}$ ) y sujetos con alto riesgo CV (p. ej., dislipidemia primaria aterogénica). Se excluyeron personas con enfermedad CV, enfermedad renal crónica y expectativa de vida a menos de un año. El estudio se llevó de acuerdo con los estatutos de la Declaración de Helsinki. Todos los participantes firmaron consentimiento informado previo a la participación del estudio. El Comité de Ética del Instituto Nacional de Ciencias Médicas Salvador Zubirán aprobó la realización de este estudio.

\section{Evaluación bioquímica, antropométrica}

Se obtuvieron muestras sanguíneas venosas después de un ayuno de 8 a 12 horas. El análisis de 
glucosa plasmática se realizó mediante un analizador automatizado (Yellow Springs Instruments, Yellow Springs, OH, EE.UU.), las concentraciones de insulina fueron medidas mediante inmunoensayo de quimioluminiscencia (Beckman Coulter Access 2), las concentraciones de $\mathrm{HbA1C}$ fueron medidas mediante cromatografía (Variant II Turbo, BIORAD) y las concentraciones del perfil de lípidos fueron medidas mediante ensayos de colorimetría (Unicel DxC 600 Synchron Clinical System Beckman Coulter). El colesterol vinculado a lipoproteínas de baja densidad (c-LDL) fue calculado de acuerdo con la fórmula de Martín ${ }^{18}$. Todos los sujetos fueron pesados utilizando básculas calibradas SECA mBCA 514 y medidos mediante estadiómetros SECA. La circunferencia de cintura fue medida mediante una cinta no elástica entre el punto medio que se intersecta del reborde costal y el borde superior de la costilla. EI IMC fue calculado dividiendo el peso en kilogramos entre la estatura en metros al cuadrado. Se utilizó el índice HOMA2-IR como el estándar para evaluar la RI. Posteriormente, se replicó la asociación mediante los subrogados QUICKI, TG/HDL, TyG, TyG*IMC y METS-IR. En la tabla 1 se muestran las fórmulas de los subrogados de estimación de RI.

\section{Evaluación de análisis de onda carotídea}

Se les pidió a los sujetos no consumir cafeína y mantenerse sin fumar las 48 horas previas. El análisis de onda carotídea fue realizado utilizando un dispositivo semiautomático (SphygmoCore XCEL, AtCor Medical Pty Ltd, EE.UU.). Al momento de la evaluación de los participantes, se les recostó en decúbito supino por 10 minutos. Se colocó un esfigmomanómetro entre el tercio proximal de la pierna derecha del paciente para registrar la onda femoral. La VOP cf y el índice de aumentación normalizado a 75 latidos por minuto (Al-75) (para reducir el efecto atribuible a la frecuencia cardiaca) fueron medidos mediante tonometría aplanadora utilizando el tiempo corregido de retardo de la onda carotídea a la onda arterial femoral ${ }^{19}$. El índice de aumentación estima el reflejo de la onda de la periferia; un regreso más temprano ocurre con la velocidad de pulso aumentado. Se definió rigidez arterial como aquellos sujetos con $\mathrm{VOP}_{\mathrm{cf}}$ arriba del percentil $90(p \geq 90)(>7.77 \mathrm{~mm} / \mathrm{s})$.

\section{Análisis estadístico}

Las variables cualitativas en forma de conteo absoluto y porcentaje. Las variables cuantitativas como
Tabla 1. Formulas de índices subrogados para estimar resistencia a la insulina. Las unidades de glucosa, TG y HDL se utilizaron en $\mathrm{mg} / \mathrm{dl}$. Las unidades de insulina fueron $\mathrm{UI} / \mathrm{ml}$

\begin{tabular}{l|l}
\hline Índice & Formula \\
\hline HOMA2-IR & https://www.dtu.ox.ac.uk/homacalculator/ \\
METS-IR & $\frac{(\operatorname{Ln}[(2 * \text { glucosa })+\text { triglicéridos }]) * I M C}{\operatorname{Ln}(\text { colesterol } H D L)}$
\end{tabular}

\section{QUICKI}

$$
\frac{1}{(\log (\text { insulina })+\log (\text { glucosa }))}
$$

TyG

$$
\operatorname{Ln}\left(\text { triglicéridos* } \frac{[\text { glucosa }]}{2}\right)
$$

TyG*IMC

$$
(T y G)^{\star}(I M C)
$$

TG/HDL

$$
\text { (Triglicéridos) / (ColesterolHDL) }
$$

HOMA2-IR: Homeostatic Model Assessment 2-Insulin Resistance; METS-IR: Metabolic score for IR; QUICKI: Quantitative Insulin Sensitivity Check Index; TyG: ratio triglicéridos/ glucosa; TyG*IMC: TyG*índice de masa corporal; TG/HDL: ratio TG/lipoproteínas de alta densidad.

media (desviación estándar) o mediana (rango intercuartil [RIQ]) según su distribución normal. Se aplicaron transformaciones logarítmicas. Se compararon los sujetos con rigidez arterial contra aquellos sin rigidez arterial utilizando las pruebas estadísticas de t de Student o U de Mann-Whitney.

\section{Correlación entre subrogados de resistencia a la insulina}

Se evaluó la correlación de HOMA2-IR y los otros subrogados con VOP $_{\text {of }}$ y el Al-75. Se ajustaron las correlaciones por edad, sexo, hipertensión arterial, tabaquismo, HbA1c, cintura y dislipidemia. Para evaluar diferencias entre sujetos con RI por nuestros subrogados, se utilizaron los puntos de corte previamente publicados por Almeda-Valdez, et al. ${ }^{20}$. Se compararon los parámetros de $\mathrm{VOP}_{\mathrm{cf}}$ entre sujetos con RI utilizando la prueba t de Student.

\section{Evaluación de puntos de corte para identificar rigidez arterial}

Se calcularon los puntos de corte para identificar rigidez arterial. Se estimó la sensibilidad, especificidad, valor predictivo positivo y negativo de cada subrogado de RI. Se obtuvo el área bajo la curva $(A B C)$ de cada 
Tabla 2. Características generales de la población de estudio

\begin{tabular}{|c|c|c|c|c|}
\hline Parámetro & $\begin{array}{l}\text { Población total } \\
\qquad(n=358)\end{array}$ & $\begin{array}{c}\text { Sin rigidez arterial } \\
(n=323)\end{array}$ & $\begin{array}{l}\text { Con rigidez arterial } \\
\qquad(n=35)\end{array}$ & Valor $p$ \\
\hline Sexo femenino (\%) & $260(72.6 \%)$ & 238 (75.1) & $18(51.4)$ & 0.001 \\
\hline Edad (años) & $50(39-57)$ & $46.6(12.7)$ & $53(7.65)$ & $<0.001$ \\
\hline Prediabetes (\%) & 254 (70.9) & 223 (70.3) & $26(74.3)$ & 0.627 \\
\hline Peso (kg) & $76.2( \pm 14.9)$ & $75.7( \pm 14.9)$ & $81.7( \pm 15.8)$ & 0.005 \\
\hline Cintura (cm) & $95.5( \pm 12.1)$ & $93.6( \pm 11.7)$ & $98.8( \pm 11.3)$ & 0.003 \\
\hline IMC (kg/m²) & $28.9(26.4-32.2)$ & $28(26-32)$ & $29.2(26.6-35)$ & 0.065 \\
\hline Glucosa (mg/dl) & $96.3( \pm 12.8)$ & $95.6( \pm 11.1)$ & $102.2( \pm 22.6)$ & 0.004 \\
\hline Triglicéridos (mg/dl) & $137(95-187)$ & $133(92-182)$ & $159(108-197)$ & 0.093 \\
\hline Colesterol total (mg/dl) & $197( \pm 43.1)$ & $194( \pm 42)$ & $188( \pm 45.8)$ & 0.239 \\
\hline Colesterol HDL (mg/dl) & $46.3( \pm 12.2)$ & $46.7( \pm 12)$ & $41.9( \pm 12)$ & 0.027 \\
\hline Colesterol LDL (mg/dl) & $123( \pm 35.5)$ & $121( \pm 35)$ & $116( \pm 35.7)$ & 0.320 \\
\hline Insulina (UI/dl) & $8.9(6-12.9)$ & $8.7(5.7-11.9)$ & $9.9(6.8-14.9)$ & 0.313 \\
\hline HbA1c (\%) & $5.8( \pm 0.75)$ & $5.8( \pm 0.60)$ & $6.3( \pm 1.18)$ & $<0.001$ \\
\hline Acido úrico (mg/dl) & $5.4( \pm 1.3)$ & $5.3( \pm 1.29)$ & $5.6( \pm 1.36)$ & 0.343 \\
\hline Creatinina (mg/dl) & $0.87( \pm 1.9)$ & $0.86( \pm 2.45)$ & $0.81( \pm 0.21)$ & 0.712 \\
\hline AST (mg/dl) & $28.2( \pm 16.2)$ & $28.3( \pm 17)$ & $27.8( \pm 8.2)$ & 0.879 \\
\hline ALT (mg/dl) & $28.3( \pm 19.9)$ & $28.3( \pm 17)$ & $30.1( \pm 15.9)$ & 0.534 \\
\hline GGT (mg/dl) & $26.4( \pm 26.2)$ & $25.9( \pm 26.8)$ & $28.9( \pm 21.7)$ & 0.550 \\
\hline APO B (mg/dl) & $112.25( \pm 28.4)$ & $109( \pm 27.7)$ & $102( \pm 29.5)$ & 0.321 \\
\hline $\mathrm{VOP}_{\mathrm{cf}}(\mathrm{mm} / \mathrm{s})$ & $6.52( \pm 1.34)$ & $5.92( \pm 0.84)$ & $8.58( \pm 0.65)$ & $<0.001$ \\
\hline Al-75 (\%) & $34.97( \pm 13.22)$ & $34.3( \pm 13.9)$ & $33.7( \pm 9.71)$ & 0.604 \\
\hline HOMA2-IR & $1.2(0.8-1.7)$ & $1.2(0.8-1.6)$ & $1.3(0.9-2.0)$ & 0.144 \\
\hline METS-IR & $45.6( \pm 9.47)$ & $45.1( \pm 9.25)$ & $48.8( \pm 10.5)$ & 0.026 \\
\hline QUICKI & $0.344( \pm 0.033)$ & $0.346( \pm 0.033)$ & $0.335( \pm 0.033)$ & 0.079 \\
\hline TyG & $8.78( \pm 0.58)$ & $8.73( \pm 0.58)$ & $8.99( \pm 0.57)$ & 0.075 \\
\hline TyG*IMC & $259.8( \pm 49.4)$ & $257.4( \pm 47.63)$ & $273.9( \pm 54.4)$ & 0.057 \\
\hline TG/HDL & $2.99(1.8-4.6)$ & $2.8(1.7-4.5)$ & $3.8(2.4-5.8)$ & 0.017 \\
\hline
\end{tabular}

subrogado. El análisis estadístico fue realizado con el lenguaje de programación $\mathrm{R}$ (Versión 3.6.1).

\section{Resultados}

Se incluyeron 358 sujetos, $260(72.6 \%)$ mujeres con una mediana de edad de 50 (RIQ: 39-57) años. Las características de la población se presentan en la tabla 2. Los sujetos con mayor rigidez arterial fueron hombres de mayor edad, con mayor peso, perímetro de cintura y con una concentración mayor de glucosa en ayuno, TG y HbA1c y con menores concentraciones de $\mathrm{c}-\mathrm{HDL}$. El valor promedio de la VOP $y$ del Al-75 en esta población fueron $6.52( \pm 1.34)$ $\mathrm{mm} / \mathrm{s}$ y $34.9( \pm 13.22) \%$, respectivamente. Con respeto a los índices subrogados (Tabla 1), los valores 

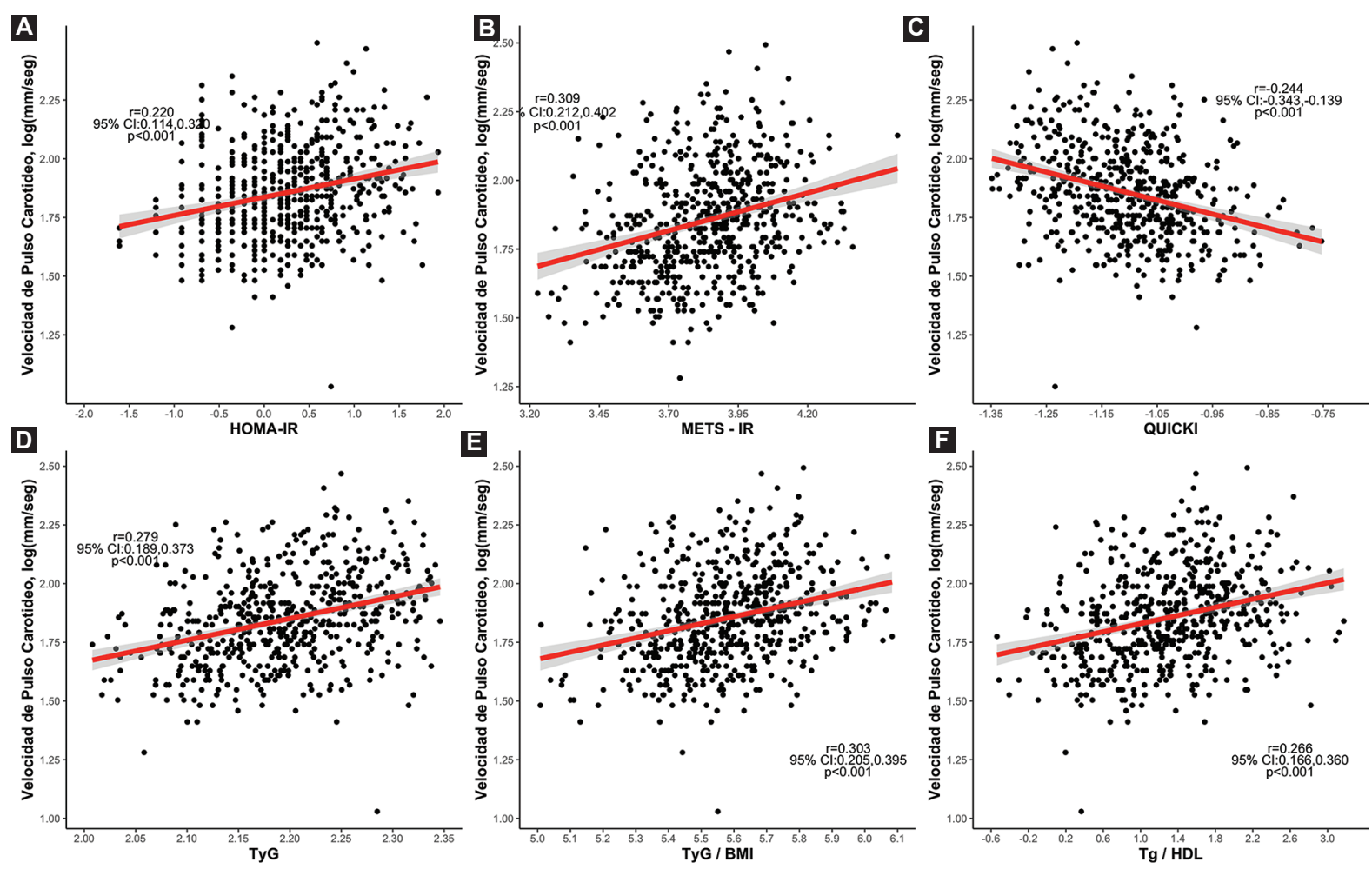

Figura 1. Correlación de subrogados de resistencia a la insulina con velocidad de pulso carotídea. Las variables fueron transformadas a su logaritmo para alcanzar distribución paramétrica.

IC 95\%: índice de confianza del 95\%; HOMA2-IR: Homeostatic Model Assessment 2-Insulin Resistance; METS-IR: Metabolic score for IR; QUICKI: Quantitative Insulin Sensitivity Check Index; TyG: ratio triglicéridos/glucosa; TyG*IMC: TyG*índice de masa corporal; TG/HDL: ratio TG/lipoproteínas de alta densidad.

promedios/medianos fueron: HOMA2-IR 1.2 (RIQ: 0.8-1.7), METS-IR $45.6( \pm 9.47)$, QUICKI $0.344( \pm$ $0.03)$, TyG $8.78( \pm 0.58)$, TyG*IMC $259.8( \pm 49.4)$ y TG/HDL 2.99 (RIQ: 1.8-4.6). Solo los índices METS-IR y TG/HDL fueron significativamente relacionados con mayor rigidez arterial

\section{Correlación de velocidad de pulso carotídeo con subrogados de resistencia a la insulina}

En el análisis de correlación bivariante, se observó una correlación con HOMA2-IR y la VOPcf ( $r$ : 0.220; intervalo de confianza del 95\% [IC 95\%]: 0.114$0.320)$ que se mantuvo al ajustar por covariables (r: 0.128; IC 95\%: 0.018-0.234). La asociación se replicó con el resto de los subrogados. La mayor correlación se observó para el índice METS-IR (r: 0.309; IC 95\%: 0.212-0.402) y el índice TyG*IMC (r: 0.279; IC 95\%: 0.189-0.373) (Fig. 1). Para Al-75, el índice TyG*IMC tuvo el mayor grado de correlación (r: 0.131; IC 95\%: 0.027-0.231). Sin embargo, al ajustar por covariables el índice HOMA2-IR mantuvo la mayor asociación con Al-75 (r: 0.168; IC 95\%: 0.060-0.272) (Tabla 3). Finalmente, los pacientes con RI tuvieron un aumento de los valores de VOP $_{\text {of }}$ evaluada por todos los subrogados (Fig. 2). Explorando los valores de $\mathrm{VOP}_{\text {cf }}$ en aquellos con prediabetes, se confirmó que esta población tuvo mayor rigidez arterial comparada con aquellos sin prediabetes (Fig. 3).

\section{Puntos de corte para identificar rigidez arterial}

Para cada subrogado se determinó el mejor punto de corte para detectar rigidez arterial. El índice HOMA2-IR mostró un adecuado rendimiento predictivo. Esta asociación fue replicada para el resto de los subrogados a RI. Los índices con mejor ABC fueron el índice METS-IR (ABC: 0.61; IC 95\%: 0.51-0.71) y el índice TyG (ABC: 0.61; IC 95\%: 0.51-0.69). El subrogado con mayor sensibilidad, con una $A B C$ significativa, fue el índice TyG (S: 0.94; IC 95\%: 
Tabla 3. Correlación de Pearson entre subrogados de resistencia a la insulina y estimación de rigidez arterial. Las variables de ajuste son: edad, sexo, tabaquismo activo, hipertensión arterial sistémica, hemoglobina glucosilada y cintura

\begin{tabular}{|c|c|c|c|c|}
\hline \multirow[t]{2}{*}{ Subrogados } & \multicolumn{2}{|c|}{$V P_{\text {of }}$} & \multicolumn{2}{|c|}{ Al-75 } \\
\hline & Bivariante & Ajustado & Bivariante & Ajustado \\
\hline HOMA2-IR & $\begin{array}{c}0.220 \\
(0.114,0.320)\end{array}$ & $\begin{array}{c}0.128 \\
(0.018,0.234)\end{array}$ & $\begin{array}{c}0.033 \\
(-0.074,0.141)\end{array}$ & $\begin{array}{c}0.168 \\
(0.060,0.272)\end{array}$ \\
\hline METS-IR & $\begin{array}{c}0.309 \\
(0.212,0.402)\end{array}$ & $\begin{array}{c}0.171 \\
(0.063,0.275)\end{array}$ & $\begin{array}{c}0.099 \\
(0.018,0.178)\end{array}$ & $\begin{array}{c}0.164 \\
(0.082-0.241)\end{array}$ \\
\hline QUICKI & $\begin{array}{c}-0.244 \\
(-0.343,-0.139)\end{array}$ & $\begin{array}{c}-0.119 \\
(-0.226,-0.01)\end{array}$ & $\begin{array}{c}-0.063 \\
(-0.145,0.019)\end{array}$ & $\begin{array}{c}-0.144 \\
(-0.225,-0.062)\end{array}$ \\
\hline TyG & $\begin{array}{c}0.279 \\
(0.189,0.373)\end{array}$ & $\begin{array}{c}0.081 \\
(-0.027,0.189)\end{array}$ & $\begin{array}{c}0.021 \\
(-0.083,0.124)\end{array}$ & $\begin{array}{c}0.126 \\
(0.018,0.232)\end{array}$ \\
\hline TyG*IMC & $\begin{array}{c}0.303 \\
(0.205,0.395)\end{array}$ & $\begin{array}{c}0.183 \\
(0.053,0.286)\end{array}$ & $\begin{array}{c}0.131 \\
(0.027,0.231)\end{array}$ & $\begin{array}{c}0.164 \\
(0.059,0.269)\end{array}$ \\
\hline TG/HDL & $\begin{array}{c}0.266 \\
(0.166,0.360)\end{array}$ & $\begin{array}{c}0.085 \\
(-0.024,0.193)\end{array}$ & $\begin{array}{c}-0.042 \\
(-0.145,0.062)\end{array}$ & $\begin{array}{c}0.118 \\
(0.009,0.223)\end{array}$ \\
\hline
\end{tabular}

VOPcf: velocidad de pulso carotídea; Al-75: índice de aumentación normalizado; HOMA2-IR: Homeostatic Model Assessment 2-Insulin Resistance; METS-IR: Metabolic score for IR; QUICKI: Quantitative Insulin Sensitivity Check Index; TyG: ratio triglicéridos/glucosa; TyG*IMC: TyG*indice de masa corporal; TG/HDL: ratio TG/lipoproteínas de alta densidad.
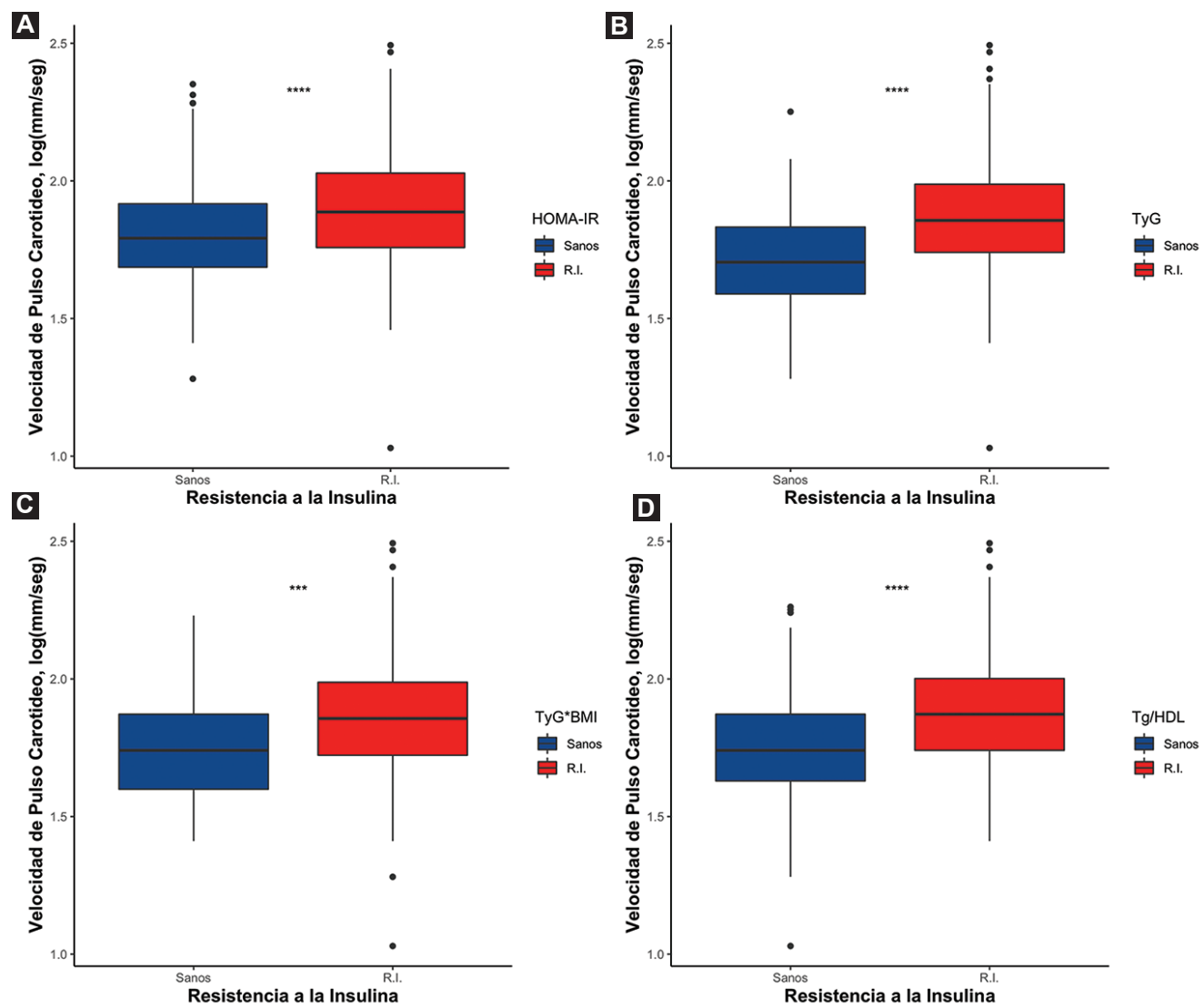

Figura 2. Diferencias de velocidad de pulso carotídeo entre sujetos con resistencia a la insulina evaluado por subrogado. ${ }^{*} p<0.05$.

$+p<0.001$

$\neq p<0.0001$.

RI: resistencia a la insulina; HOMA2-IR: Homeostatic Model Assessment 2-Insulin Resistance; TyG: ratio triglicéridos/glucosa; TyG*IMC: TyG*índice de masa corporal; TG/HDL: ratio TG/lipoproteínas de alta densidad. 

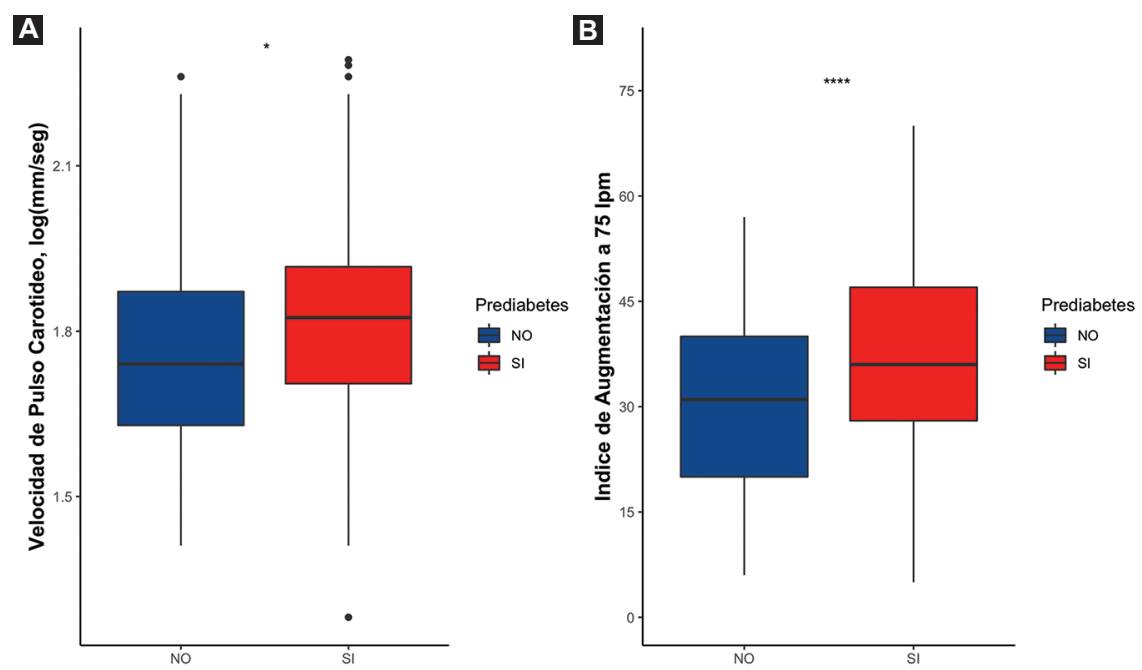

Figura 3. Diferencias de velocidad de pulso carotídeo (A) y el índice de aumentación a 75 lpm (B) entre sujetos con y sin prediabetes. ${ }^{*} p<0.05$.

${ }^{t} p<0.001$.

${ }^{\ddagger} p<0.0001$.

Tabla 4. Puntos de corte para identificar rigidez arterial $\left(\mathrm{VOP}_{\mathrm{cf}} \mathbf{p} \geq 90\right)$ en sujetos sanos. Rigidez arterial se definió como aquellos sujetos con VOP ${ }_{c f}$ encima del percentil $90(p \geq 90)$ con un valor de 7.77

\begin{tabular}{|c|c|c|c|c|c|c|}
\hline Variables & Punto de corte & Sensibilidad (\%) & Especificidad (\%) & VPP (\%) & VPN (\%) & $\mathrm{ABC}$ \\
\hline HOMA2-IR & 1.7 & $\begin{array}{c}0.43 \\
(0.26-0.62)\end{array}$ & $0.76(0.71-0.81)$ & $\begin{array}{c}0.17 \\
(0.14-0.30)\end{array}$ & $\begin{array}{c}0.92 \\
(0.85-0.94)\end{array}$ & $\begin{array}{c}0.59 \\
(0.48-0.69)\end{array}$ \\
\hline METS-IR & 41.6 & $\begin{array}{c}0.82 \\
(0.66-0.93)\end{array}$ & $\begin{array}{c}0.39 \\
(0.33-0.48)\end{array}$ & $\begin{array}{c}0.13 \\
(0.11-0.31)\end{array}$ & $\begin{array}{c}0.95 \\
(0.89-0.96)\end{array}$ & $\begin{array}{c}0.61 \\
(0.51-0.71)\end{array}$ \\
\hline QUICKI & 0.39 & $\begin{array}{c}0.13 \\
(0.04-0.29)\end{array}$ & $\begin{array}{c}0.91 \\
(0.88-0.94)\end{array}$ & $\begin{array}{c}0.14 \\
(0.10-0.32)\end{array}$ & $\begin{array}{c}0.91 \\
(0.71-0.94)\end{array}$ & $\begin{array}{c}0.40 \\
(0.29-0.51)\end{array}$ \\
\hline TyG & 8.4 & $\begin{array}{c}0.94 \\
(0.81-0.99)\end{array}$ & $\begin{array}{c}0.27 \\
(0.22-0.32)\end{array}$ & $\begin{array}{c}0.27 \\
(0.10-0.55)\end{array}$ & $\begin{array}{c}0.98 \\
(0.92-0.98)\end{array}$ & $\begin{array}{c}0.61 \\
(0.51-0.70)\end{array}$ \\
\hline TyG*IMC & 301.9 & $\begin{array}{c}0.34 \\
(0.19-0.52)\end{array}$ & $\begin{array}{c}0.84 \\
(0.79-0.88)\end{array}$ & $\begin{array}{c}0.19 \\
(0.15-0.33)\end{array}$ & $\begin{array}{c}0.92 \\
(0.84-0.94)\end{array}$ & $\begin{array}{c}0.59 \\
(0.48-0.69)\end{array}$ \\
\hline $\mathrm{TG} / \mathrm{HDL}$ & 3.05 & $\begin{array}{c}0.69 \\
(0.51-0.83)\end{array}$ & $\begin{array}{c}0.52 \\
(0.47-0.58)\end{array}$ & $\begin{array}{c}0.14 \\
(0.11-0.27)\end{array}$ & $\begin{array}{c}0.94 \\
(0.88-0.95)\end{array}$ & $\begin{array}{c}0.60 \\
(0.51-0.70)\end{array}$ \\
\hline
\end{tabular}

VOP $_{c f}$ : velocidad de pulso carotídea; VPP: valor predictivo positivo; VPN: valor predictivo negativo; ABC: área bajo la curva; HOMA2-IR: Homeostatic Model Assessment 2-Insulin

Resistance; METS-IR: Metabolic score for IR; QUICKI: Quantitative Insulin Sensitivity Check Index; TyG: ratio triglicéridos/glucosa; TyG*IMC: TyG*índice de masa corporal; TG/HDL: ratio TG/lipoproteínas de alta densidad.

0.81-0.99), mientras que el índice TyG* ${ }^{*} M C$ tuvo la mejor especificidad (E: 0.84; IC 95\%: 0.79-0.88) con una $A B C$ significativa (Tabla 4).

\section{Discusión}

Se evaluó la asociación entre la RI y la velocidad de la onda del pulso en una población mexicana sin DT2. Los pacientes con RI, evaluada por subrogados, tuvieron un aumento en los valores de $\mathrm{VOP}_{\mathrm{cf}}$ independiente de factores de riesgo. Los índices con mayor grado de asociación fueron METS-IR y TyG*IMC, y los con mejor $A B C$ para detectar rigidez arterial fueron METS-IR y TyG. Los sujetos con prediabetes tuvieron una mayor rigidez arterial comparada con aquellos sin prediabetes.

Se ha mostrado una relación directa entre la $\mathrm{VOP}_{c f}$ y la aterosclerosis, y en estudios longitudinales este parámetro es un marcador de riesgo CV independiente ${ }^{21}$. Un aumento en la rigidez arterial puede ser uno de los mecanismos que explica la asociación entre el riesgo CV y la RI. Estudios epidemiológicos 
han mostrado que la RI es un factor independiente para el desarrollo de la rigidez arterial. En el estudio ARIC, las personas con DT2 o intolerancia a los carbohidratos tuvieron arterias más rígidas que personas con normoglucemia ${ }^{22}$. Se especuló que el efecto de glucosa, insulina y TG en conjunto juegan un papel en el desarrollo de la rigidez arterial. Se han mostrado diferencias significativas en la VOP entre sujetos con y sin síndrome metabólico ${ }^{23}$. Los mecanismos específicos involucrados no son claros, pero sabemos que la RI tiene efectos directos e indirectos sobre la vasculatura. La hiperinsulinemia puede aumentar el tono simpático, activar el sistema renina-angiotensina, estimular inflamación vascular y reducir la vasodilatación dependiente del endotelio mediado por flujo. El resultado final es disfunción endotelial y una respuesta vasomotora inadecuada al endotelio proinflamatorio y procoagulante, aumentado el riesgo para rigidez arterial ${ }^{24}$.

Varios autores han evaluado la asociación entre los subrogados de la RI y la rigidez arterial. En personas sin enfermedad CV, se ha confirmado una asociación significativa entre TyG y VOP ${ }_{c f}{ }^{25}$. El HOMA2-IR fue independientemente asociado con $\mathrm{VOP}_{\mathrm{cf}}$ en personas sin diabetes o con prediabetes ${ }^{26,27}$. Webb, et al. evaluaron la $\mathrm{VOP}_{\text {cf en }}$ personas con normoglucemia, regulación de glucosa anormal (glucosa en ayuno anormal y/o intolerancia a los carbohidratos) y DT2 ${ }^{28}$. En los últimos dos grupos hubo una asociación significativa con la rigidez arterial. La intolerancia a la glucosa y HOMA2-IR fueron los predictores independientes más importantes para la rigidez arterial.

Este estudio tiene algunas limitaciones. Se utilizaron subrogados para RI en plazo del estándar de oro. La población fue heterogénea y puede ser no representativa de la población general. La mayoría fueron mujeres, representando un potencial sesgo de selección. No se obtuvieron datos sobre ejercicio y de medicamentos que podrían influir el proceso de aterosclerosis y la $\mathrm{RI}$.

\section{Conclusiones}

Es el primer estudio en población mexicana que confirma la relación entre la RI y la rigidez arterial en personas sin diabetes. Se corrobora que la relación entre la RI y la rigidez arterial está presente desde etapas no-diabéticas; esta asociación puede conllevar mayor riesgo CV en el futuro. El índice HOMA2-IR mostró una asociación que se replicó con los diversos subrogados de RI. El mejor desempeño para detectar la rigidez arterial fue con los índices METS-IR y TyG. Estos son parámetros sencillos para calcular y pueden ser incluidos en la evaluación del riesgo CV.

\section{Agradecimientos}

Neftali Eduardo Antonio-Villa y Arsenio VargasVázquez pertenecen al programa MD/PhD PECEM de la Facultad de Medicina de la UNAM. Neftali Eduardo Antonio-Villa y Arsenio Vargas-Vázquez son apoyados por CONACyT.

\section{Financiamiento}

La presente investigación no ha recibido ninguna beca específica de agencias de los sectores públicos, comercial o sin ánimo de lucro.

\section{Conflicto de intereses}

Los autores declaran que no tienen conflicto de intereses.

\section{Responsabilidades éticas}

Protección de personas y animales. Los autores declaran que para esta investigación no se han realizado experimentos en seres humanos ni en animales.

Confidencialidad de los datos. Los autores declaran que han seguido los protocolos de su centro de trabajo sobre la publicación de datos de pacientes.

Derecho a la privacidad y consentimiento informado. Los autores han obtenido el consentimiento informado de los pacientes y/o sujetos referidos en el artículo. Este documento obra en poder del autor de correspondencia.

\section{Bibliografía}

1. Howard G, O'Leary DH, Zaccaro D, Haffner S, Rewers M, Hamman R, et al. Insulin sensitivity and atherosclerosis. Circulation. 1996;93:1809-17.

2. Adeva-Andany MM, Martínez-Rodríguez J, González-Lucán M, Fernández-Fernández C, Castro-Quintela E. Insulin resistance is a cardiovascular risk factor in humans. Diabetes Metab Syndr. 2019;13(2):1449-55.

3. Poon AK, Meyer ML, Tanaka H, Selvin E, Pankow J, Zeng D, et al. Association of insulin resistance, from mid-life to late-life, with aortic stiffness in late-life: the Atherosclerosis Risk in Communities Study. Cardiovasc Diabetol. 2020;19:11.

4. Fu S, Lin Y, Luo L, Ye P. Relationship between central arterial stiffness and insulin resistance in Chinese community-dwelling population without diabetes mellitus. Int J Endocrinol. 2017;2017:1073919.

5. Cruickshank K, Riste L, Anderson SG, Wright JS, Dunn G, Gosling RG. Aortic pulse-wave velocity and its relationship to mortality in diabetes and glucose intolerance: an integrated index of vascular function? Circulation. 2002;106(16):2085-90.

6. Gajdova J, Karasek D, Goldmannova D, Krystynik O, Schovanek J, Vaverkova $\mathrm{H}$, et al. Pulse wave analysis and diabetes mellitus. A systematic review. Biomed Pap Med Fac Univ Palacky Olomouc Czech Re- 
pub. 2017;161(3):223-33.

7. Janić $M$, Lunder M, Sabovič M. Arterial stiffness and cardiovascular therapy. Biomed Res Int. 2014;2014:621437.

8. Vlachopoulos C, Aznaouridis K, Stefanadis C. Prediction of cardiovascular events and all-cause mortality with arterial stiffness: a systematic review and meta-analysis. J Am Coll Cardiol. 2010;55:1318-27.

9. Ben-Shlomo Y, Spears M, Boustred C, May M, Anderson SG, Benjamin EJ et al. Aortic pulse wave velocity improves cardiovascular event prediction: an individual participant meta-analysis of prospective observational data from 17,635 subjects. J Am Coll Cardiol. 2014;63:636-46.

10. Faconti L, Nanino E, Mills CE, Cruickshank KJ. Do arterial stiffness and wave reflection underlie cardiovascular risk in ethnic minorities? JRSM Cardiovasc Dis. 2016;5:1-9.

11. Defronzo R, Tobin J, Andres R. Glucose clamp technique: a method for quantifying insulin secretion and resistance. Am J Physiol. 1979:237:E214-23.

12. Otten J, Ahrén B, Olsson T. Surrogate measures of insulin sensitivity vs the hyperinsulinaemic-euglycaemic clamp: a meta-analysis. Diabetologia. 2014;57(9):1781-8.

13. Fiorentino TV, Marini MA, Succurro E, Andreozzi F, Sest G. Relationships of surrogate indexes of insulin resistance with insulin sensitivity assessed by euglycemic hyperinsulinemic clamp and subclinical vascular damage. BMJ Open Diabetes Res Care. 2019;7(1):e000911.

14. Borai A, Livingstone C, Kaddam I, Ferns G. Selection of the appropriate method for the assessment of insulin resistance. BMC Med Res Methodol. 2011:11:158-67.

15. Bello-Chavolla OY, Almeda-Valdes $P$, Gomez-Velasco D, Viveros-Ruiz T, Cruz-Bautista I, Romo-Romo A, et al. METS-IR, a novel score to evaluate insulin sensitivity, is predictive of visceral adiposity and incident type 2 diabetes. Eur J Endocrinol. 2018:178(5):533-44.

16. Townsend RR, Wilkinson IB, Schiffrin EL, Avolio AP, Chirinos JA, Cockcroft JR, et al. Recommendations for improving and standardizing vascular research on arterial stiffness: a scientific statement from the American Heart Association. Hypertension. 2015;66:698-722.

17. Townsend RR. Arterial stiffness: Recommendations and standardization. Pulse. 2016;4(suppl 1):3-7.

18. Martin SS, Blaha MJ, Elshazly MB, Toth PP Kwiterovich PO Blumenthal RS, et al. Comparison of a novel method vs the Friedewald equation for estimating low-density lipoprotein cholesterol levels from the standard lipid profile. J Am Med Assoc. 2013;310:2061-8.

19. Van Bortela LM, Laurent $S$, Boutouyrie $P$, Chowienczyk $P$, Cruickshank JK, De Backera T, et al.; on behalf of the Artery Society, the European Society of Hypertension Working Group on Vascular Structure and Function and the European Network for Noninvasive Investigation of Large Arteries. Expert consensus document on the measurement of aortic stiffness in daily practice using carotid-femoral pulse wave velocity. J Hypertension. 2012;30:445-8

20. Almeda-Valdés P, Bello-Chavolla OY, Caballeros-Barragán CR, Gómez-Velasco DV, Viveros-Ruiz T, Vargas-Vázquez A, et al. Índices para la evaluación de la resistencia a la insulina en individuos mexicanos sin diabetes. Gac Med Mex. 2018:154(Supp 2):S50-S55.

21. Kim HL, Kim SH. Pulse wave velocity in atherosclerosis. Front Cardiovasc Med. 2019;6:41.

22. Salomaa V, Riley W, Kark JD, Nardo C, Folsom AR. Non-insulin-dependent diabetes mellitus and fasting glucose and insulin concentrations are associated with arterial stiffness indexes. The ARIC Study. Atherosclerosis Risk in Communities Study. Circulation. 1995;91(5):1432-43.

23. Peñaherreraa CA, Peñaherrerab R, Duartea MC, Peñaherreraa E. Assessment of arterial stiffness in patients with metabolic syndrome in Ecuador: A cross-sectional study. Diabetes Metab Syndr. 2017;11(3):199-202.

24. Novo G, Manno G, Russo R, Buccheri D, Dell'Oglio S, Morreale P, et al. Impact of insulin resistance on cardiac and vascular function. Int J Cardiol. 2016;221:1095-9

25. Won KB, Park GM, Lee SE, Cho IJ, Kim HC, Lee BK, et al. Relationship of insulin resistance estimated by triglyceride glucose index to arterial stiffness. Lipids Health Dis. 2018;17(1):268

26. Fu S, Lin Y, Luo L, Ye P. Relationship between Central Arterial Stiffness and Insulin Resistance in Chinese Community-Dwelling Population without Diabetes Mellitus. Int J Endocrinol. 2017;2017:1073919.

27. Ho CT, Lin CC, Hsu HS, Liu CS, Davidson LE, Li TC, et al. Arterial stiffness is strongly associated with insulin resistance in Chinese - A population-based study (Taichung Community Health Study, TCHS). J Atheroscler Thromb. 2011;18 (2):122-30.

28. Webb DR, Khunti K, Silverman R, Gray LJ, Srinivasan B, Lacy PS, et al. Impact of metabolic indices on central artery stiffness: independent association of insulin resistance and glucose with aortic pulse wave velocity. Diabetologia. 2010;53:1190-8. 